\title{
Cluster dynamics in dense granular gases of rod-like particles
}

\author{
Dmitry Puzyrev ${ }^{1, *}$, Raúl Cruz Hidalgo ${ }^{2}$, David Fischer ${ }^{1}$, Kirsten Harth $^{1}$, Torsten Trittel ${ }^{1}$, and Ralf Stannarius ${ }^{1}$ \\ ${ }^{1}$ Institute of Physics, Otto von Guericke University, Magdeburg, Germany \\ ${ }^{2}$ Física y Matemática Aplicada, Facultad de Ciencias, Universidad de Navarra, Pamplona, Spain
}

\begin{abstract}
Granular gases are interesting multiparticle systems which, irrespective of the apparent simplicity of particle interactions, exhibit a rich scenario of so far only little understood features. We have numerically investigated a dense granular gas composed of frictional spherocylinders which are excited mechanically by lateral vibrating container walls. This study was stimulated by experiments in microgravity on parabolic flights. The formation of spatial inhomogeneities (clusters) was observed in a region near the corners of the container, about halfway from the excitation plates. The particles in the clusters show a tendency to align parallel to the container walls, seemingly increasing the stabilizing effect of friction. The simulation results provide hints that the phase difference of the vibrations of the two excitation walls might affect the cluster dynamics.
\end{abstract}

\section{Introduction}

Granular gases have been investigated numerically in numerous studies, primarily with focus on hard sphere ensembles $[1,2]$. Their experimental realization and observation is challenging, especially in three-dimensional (3D) geometries. Gravitational forces usually mask any grain interactions unless the ensemble is in microgravity $(\mu g)$. From monoscopic or stereoscopic observations, particle positions and velocities need to be extracted, which is often possible only in very dilute systems, not in clusters or denser ensembles. All these problems implicate that experimental data are rare, even though there has been continuous progress in $\mu g$ experiments with 3D granular gases, e. g. [3-9]. For a selective and purposeful choice of experimental parameters, simulations of such ensembles in close relation to experiments are very helpful.

It has been shown $[4-6,10]$, that the choice of nonspherical, rod-like particles offers several advantages, viz. a better visibility of background particles, higher collision rates, and the improved visibility of rotational motions. Collisions of non-spherical objects may be better suited to reflect the behavior of realistic, irregular grains. While these advantages are beneficial in experiments, computation is considerably more complicated than for spheres [11-13]. Here, we present simulations of granular ensembles of spherocylinders under strong mechanical excitation in $\mu g$. The packing fractions are chosen such that the system is at the brink of forming cluster structures. Two lateral walls are vibrated for the excitation of the granulate, so that 'hot' (high kinetic energy) particles are rebounded from these side walls and form a dilute, 'gaseous' state near the walls. In some distance from the excitation walls, a condensed cluster can form with dominating permanent contacts between neighboring grains.

*e-mail: dmitry.puzyrev@ ovgu.de

A video is available at https://doi.org/10.48448/1tg1-2s71
This state can be considered as a liquid state, with colder (less kinetic energy) particles, so that the complete ensemble reflects a granular Leidenfrost phenomenon in microgravity. The dynamic phenomena and regime maps are known for spherical grains, corresponding results are reported, e.g., in Refs. [14-18]. For rods, we expect a more complex behaviour and the actual regimes are a priori unknown. Our simulations are motivated by the VIP-GRAN experiment performed on parabolic flights by the SPACE GRAINS team $[7,8]$, where we currently study clustering in ensembles of cylindrical grains.

\section{Model system}

Figure 1 shows a sketch of the simulated setup. The container possesses a square cross-section of $30 \times 30 \mathrm{~mm}^{2}$, and two opposing movable side walls (pistons) with a mean distance $L_{x}=40 \mathrm{~mm}$ at rest. Agitation of the system is executed by these two walls, performing sinusoidal vibrations with an amplitude $A=4 \mathrm{~mm}$ at a frequency of $15 \mathrm{~Hz}$, corresponding to a maximum acceleration of $\approx 3.6 \mathrm{~g}$. The phase shift $\varphi$ between the walls can be varied. In this study, we are investigating the effects of in-phase $(\varphi=0)$ and anti-phase $(\varphi=\pi)$ excitation, as well as the case of $\varphi=\pi / 2$.

We use a hybrid GPU-CPU implementation of discrete element modelling [12,13], adapted to simulate confined systems with moving walls. It solves the dynamics of a monodisperse ensemble of spherocylinders, with length $\ell$ and radius $r$, i.e. aspect ratio $\zeta=\ell / 2 r$. The contact detection between two spherocylinders reduces to finding the closest point between two line segments. Thus, the overlap distance $\delta$ results from the overlap of two spheres of radius $r$. For the particle-wall collisions, we use the same model but assume the interaction of a spherocylinder with an infinite plane. The force $\vec{F}_{i j}$ exerted on particle $i$ by 
the particle $j$ reads $\vec{F}_{i j}=-\vec{F}_{j i}=F^{\mathrm{n}} \cdot \vec{n}+F^{\mathrm{t}} \cdot \vec{t}$, where $F^{\mathrm{n}}$ is the component normal to the contact plane and $F^{\mathrm{t}}$ acts in the tangential direction. In our calculations, $F^{\mathrm{n}}$ and $F^{\mathrm{t}}$ are computed using a non-linear Hertz-Mindlin approach [19]. To mimic steel particles, the particle density $\rho_{p}=7850 \mathrm{~kg} / \mathrm{m}^{3}$ and Young modulus $Y=1 \mathrm{GPa}$ ( $Y_{w}=6 \mathrm{GPa}$ for walls) were used. Note that presupposing a constant restitution coefficient is not valid when executing DEM of non-spherical particles, due to the dependence of the energy loss on the collision details. However, the energy loss still can be quantified by an effective normal restitution coefficient $e_{n}$, from which the collision damping parameter $\beta$ of the model is obtained, $\beta=\frac{\ln e_{n}}{\sqrt{\ln e_{n}{ }^{2}+\pi^{2}}}$. Thus, we fixed $e_{n}=0.85$ and friction coefficient $\mu=0.4$ (particle-particle and wall-particle), and simulation time step $\Delta t=1 / 18 \mu$ s [20].

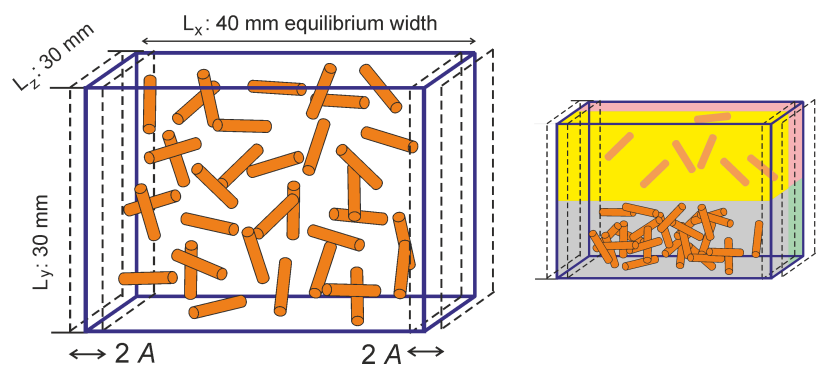

Figure 1. Left: Sketch of the setup for the simulations. It is similar to the VIP-GRAN instrument described in Ref. [8]). The side walls can be vibrated to excite the particle ensemble mechanically in microgravity. Right: Sketch of the quadrants introduced in Sec. 3.1, with one cluster in the grey quadrant.

\section{Main results}

For small filling fractions $(\phi<2 \%)$, one observes the rod ensemble in a gaseous state $[7,17,21]$. For $\phi \approx 2-3 \%$, small clusters of particles start to form. Those clusters are characterized by a larger local particle number density, as well as a lower granular temperature than in the surrounding gaseous medium. The particular values of $\phi_{c}$ corresponding to the gas-cluster transition and their dependence on system parameters is of great interest $[7,21]$ and will be explored for the ensembles of rods in future studies. For larger packing fractions $(\phi>4 \%)$, a single large cluster is formed in the center of the container. It occupies most of the cross-section in the direction perpendicular to the excitation axis $x$. Similar observations were made in experiments and simulations of shaken ensembles of spherical grains [7, 21]. Here, we focus on the packing fraction $\phi=3 \%$ ( $N=544$ particles $)$, which corresponds to a system slightly above the gas cluster-transition.

\subsection{Cluster geometry and dynamics}

For the different excitation phase shifts $\varphi=\{0, \pi / 2, \pi\}$ considered here, we observe a couple of common cluster shapes which the particles roughly assume. Two examples for in-phase and anti-phase wall movement are shown in
Figure 2. One common trend that is observed for all excitation phase shifts is that the formation of clusters starts near the corners of the container parallel to the excitation axis $\vec{x}$ (where the $y$ and $z$-walls intersect). After 100 excitation periods, considerably stable cluster structures in the vicinity of those edges are observed. The shapes of those clusters roughly resemble tetrahedra. We observe that for in-phase excitation at a given packing fraction, the most stable configuration is a large cluster with a smaller adjacent one on the opposite edges of one of the nonvibrating walls, see Fig. 2, left. For the anti-phase (symmetric in $x$ ) excitation we rather observe the existence of two similar-sized clusters (see Fig. 2, right). Clustering near container walls, in particular in corner regions, is a common observation in other experiments and simulations, e.g. Refs. [17, 22]. It is related to an increased dissipation by collisions with the walls.

The reason behind these particular shapes of the clusters requires additional investigations. It seems that the particle-wall friction plays a large role in the formation of the clusters, namely, the cluster starts to form in adjacency of the corners because collisions with any of the 2 nearby walls is highly probable here, so that the mean dissipation of energy by particle-wall collisions is highest in this part of the container. One may hypothesize that the particleparticle friction then additionally stabilizes the clusters. In particular, since the particles tend to align their long axes parallel to each other in the clusters, the contact regions increase.

To check this, we performed simulations with negligible friction, $\mu=0.01$, and considerably smaller restitution coefficient $\epsilon_{n}=0.75$ : While some local temporary clustering and some increase of density in the center of container took place, stable cluster structures as seen in Fig. 2 were not observed. In this case, the local average densities calculated in similar way as in Fig. 2 stay below 100 particles $/ \mathrm{cm}^{3}$ and never reach the densities observed inside the clusters in frictional system.

We also observed that in the system with friction, the clusters tend to arise closer to the center of the container in $x$-direction. There, the energy input transferred by the particles after the collision with the vibrating walls is minimal. We performed a crude preliminary analysis of cluster movement: The container was separated into 3D "quadrants" by $y=0$ and $z=0$ planes (see Fig. 1). Since the clusters are observed near the edges of the container, each cluster is contained in one of the quadrants. Then, the cluster motion can be approximated by the motion of the center of mass $x_{m}$ of all particles in the respective quadrant. In both cases, $\varphi=0$ and $\varphi=\pi$, cluster centers slowly drift close to the midplane of the container in $x$-direction, with the amplitude of motion roughly in the range between $0.05 L_{x}$ to $0.1 L_{x}$. The motion seems to be slightly faster for in-phase excitation, $\varphi=0$. The preliminary analysis suggests a certain periodicity in this motion, with periods between the local extrema of $x_{m}$ in a range of $\approx 10$ to 20 periods of the wall vibration.

Preliminary results also indicate that the excitation phase shift of $\varphi=\pi / 2$ leads to a lower stability of the clusters than for $\varphi=0$ and $\varphi=\pi$. While still two clusters 

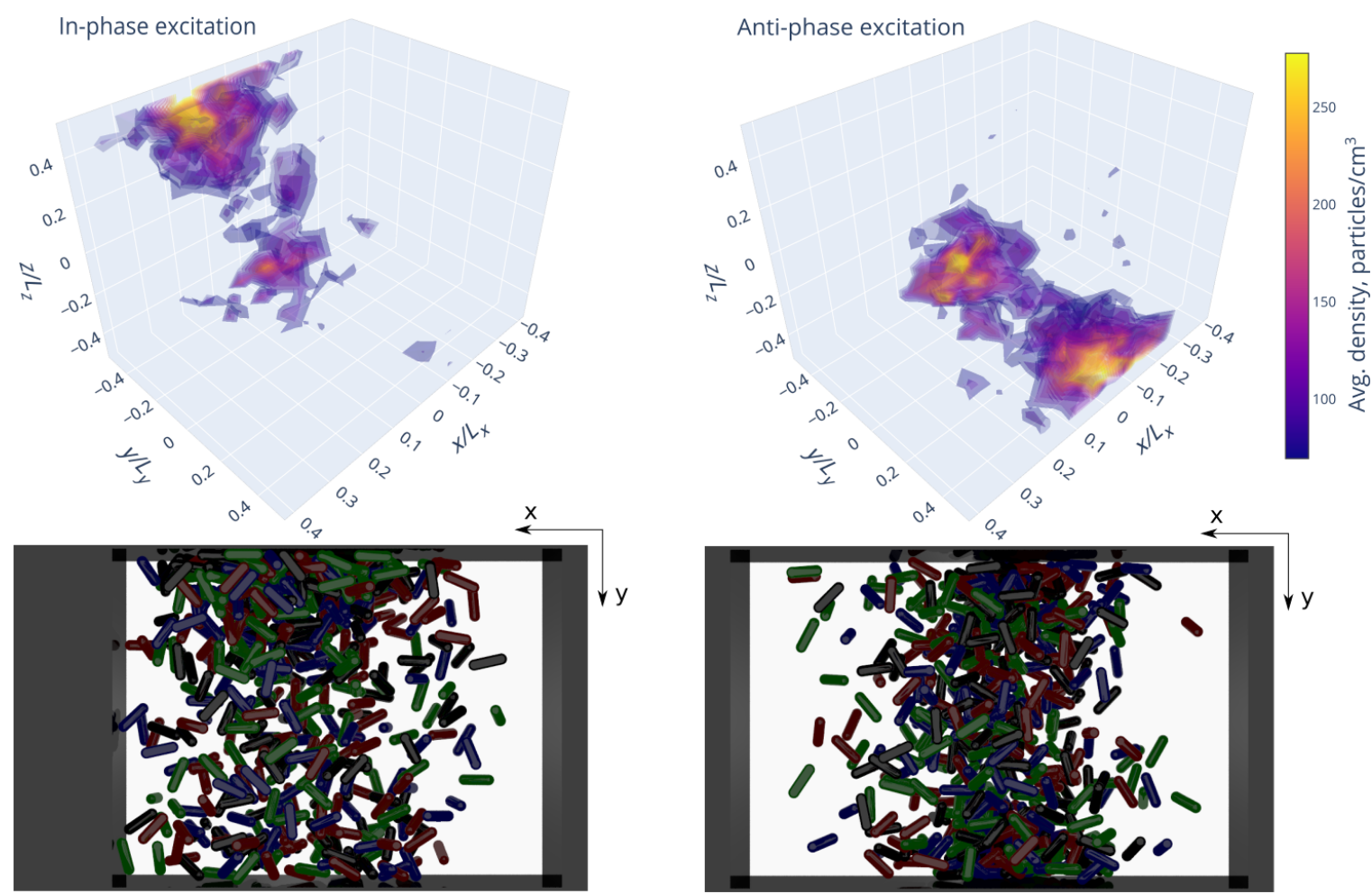

Figure 2. Isodensity surface plots for the simulated ensembles of 544 frictional rods. Density is averaged over 3 excitation periods. Left: in-phase excitation, right: anti-phase excitation. The formation of clusters is observed near the edges of the container. Below the $3 \mathrm{D}$ plots, the rendered simulation data of the respective ensembles are shown from the top view perspective.
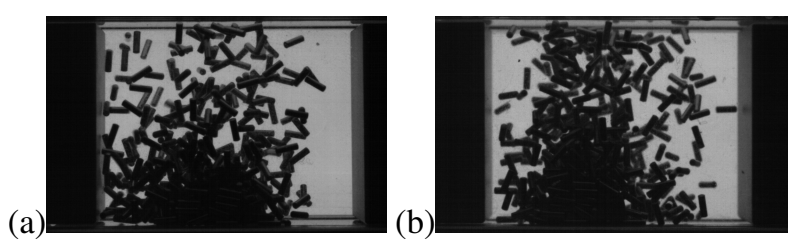

Figure 3. Exemplary experimental snapshots of front (a) and bottom (b) views showing 550 rods of $\ell=3 \mathrm{~mm}$ length (filling fraction $\phi=3 \%)$ with in-phase excitation $(\varphi=0)$. A cluster of particles near one the container edges is observed, visually resembling the simulation results from Fig. 2. Experimental data were kindly provided by the SPACE GRAINS team.

are observed, even after 150 excitation periods, they seem to constantly change their relative sizes and their centers of mass wander in $x$-direction. One of the clusters may grow substantially while the other one sometimes almost dissolved into a gaseous state. The influence of the excitation phase shift $\varphi$ will be studied in more detail in future work.

\subsection{Observation in the VIP-GRAN experiment}

Our numerical results of cluster shapes in the simulated systems (Fig. 2) can be compared to experimental images obtained under microgravity during parabolic flights by the SPACE GRAINS team, shown in Fig. 3. There, a similar system geometry and filling fraction leads to the formation of clusters near the walls of the container which visually resemble the shapes observed in the simulations. So far, there is no reliable way to reconstruct the 3D local density data from the experimental videos. The possibility of such a reconstruction, e.g., by means of machine learning techniques [23] could be envisioned as an aim of future research.

It is worth noting that the accumulation of particles near the walls and the wandering of clusters have been previously observed in experiments. These phenomena were attributed essentially to $g$-jitter (residual acceleration) rather than to the effects of the particle-wall and particle-particle friction. In our simulations, we observe those effects without inclusion of gravity or any other acceleration.

\section{Conclusions and outlook}

We have investigated dense granular gases of rod-like particles by means of numerical simulations of ensembles of frictional spherocylinders. The packing fraction was chosen just above the supposed gas-cluster transition criterion, and indeed, the formation of clusters was observed. These clusters start forming near the corners of the container about halfway between the excitation plates, similar to what has been observed for spherical grains before [17]. Rods in those denser regions show a tendency to align with their long axes parallel to each other, and parallel to the container walls. We suppose that these larger contact surfaces increase the stabilizing effect of friction in such denser assemblies of particles. Indeed, performing a sim- 
ulation with particles of very low friction coefficient, we found that clusters in that case have a much lower stability. For our rodlike grains, in particular for frictional contacts, shape effects may add additional stability to the clustered region against removal of individual grains from its surface.

Previous experiments and simulations studying the gas-to-cluster transition with spherical grains focused on in-phase (e.g. Sack et al. [24]) or anti-phase (e.g. [7]) motion of the exciting walls. Here, we present first indications that other phase angles between the two pistons' motions clearly affect the cluster dynamics.

More thorough investigations of the clustering process, the influence of the friction coefficient, the excitation and particle size parameters and also the phase of the driving are necessary to gain understanding of dynamical clustering in granular gases in particular for non-spherical grains. Due to the limited availability of microgravity experiment time, and the limited options to vary the particles' properties, realistic and validated numerical simulations are the ideal means to provide new insight. The particular roles of energy dissipation by the normal restitution coefficient and by friction need to be discriminated.

One problem of experiments in parabolic flights is the low quality of microgravity, whose influences need to be distinguished carefully from intrinsic granular dynamics. In simulations, we can include residual accelerations in two different ways: First, one can generate acceleration protocols that mimic typical $g$-jitter in the airplane in order to analyse the consequences on the grain dynamics. This allows to identify artifacts in experimental data attributed to poor microgravity quality. Second, in a more challenging simulation, one may incorporate the actual $g$ jitter data in the experiments, which are available from the flight protocols. Then one can simulate particle ensembles under the actual flight conditions. Thus, it may be possible to separate external disturbances from potential genuine effects of the granular gas. This may allow us to identify dynamic phenomena that are partially masked by $g$-jitter in parabolic flight experiments.

\section{Acknowledgements}

The authors are indebted to the SPACE GRAINS experimental team, particularly M. Noirhomme, N. Vandewalle, and E. Falcon, for providing a comprehensive data base and for unpublished footage of parabolic flight experiments. DLR is acknowledged for funding within projects Equipage II (50WM1842) and EVA (50WM2048). R.C. Hidalgo acknowledges Projects No. FIS2017-84631-P, MINECO/AEI/FEDER, UE.

\section{References}

[1] T. Pöschel, S. Luding, eds., Granular gases, Lecture Notes in Physics (Springer, Berlin, 2001), ISBN 9783-540-44506-7
[2] T. Pöschel, N.V. Brilliantov, eds., Granular gas $d y$ namics, Lecture Notes in Physics (Springer, Berlin, 2003), ISBN 978-3-540-20110-6

[3] E. Falcon, R. Wunenburger, P. Évesque, S. Fauve, C. Chabot, Y. Garrabos, D. Beysens, Phys. Rev. Lett. 83, 440 (1999)

[4] K. Harth, U. Kornek, T. Trittel, U. Strachauer, S. Höme, K. Will, R. Stannarius, Phys. Rev. Lett. 110, 144102 (2013)

[5] K. Harth, T. Trittel, K. May, S. Wegner, R. Stannarius, Adv. Space Res. 55, 1901 (2015)

[6] K. Harth, T. Trittel, S. Wegner, R. Stannarius, Phys. Rev. Lett. 120, 214301 (2018)

[7] M. Noirhomme, A. Cazaubiel, A. Darras, E. Falcon, D. Fischer, Y. Garrabos, C. Lecoutre-Chabot, S. Merminod, E. Opsomer, F. Palencia et al., Europhys. Lett. 123, 14003 (2018)

[8] S. Aumaitre, R.P. Behringer, A. Cazaubiel, E. Clément, J. Crassous, D.J. Durian, E. Falcon, S. Fauve, D. Fischer, A. Garcimartín et al., Rev. Sci. Instr. 89, 075103 (2018)

[9] P. Yu, M. Schröter, M. Sperl, Phys. Rev. Lett. 124, 208007 (2020)

[10] K. Harth, T. Trittel, U. Kornek, S. Höme, K. Will, U. Strachauer, R. Stannarius, AIP Conf. Proc. 1542, 807 (2013)

[11] F. Villemot, J. Talbot, Granular Matter 14, 91 (2012)

[12] S.M. Rubio-Largo, P.G. Lind, D. Maza, R.C. Hidalgo, Comput. Part. Mech. 2, 127 (2015)

[13] S.M. Rubio Largo, F. Alonso-Marroquin, T. T. Weinhart, S. Luding, R.C. Hidalgo, Physica A 443, 477 (2016)

[14] E. Opsomer, F. Ludewig, N. Vandewalle, Phys. Rev. E 84, 051306 (2011)

[15] E. Opsomer, F. Ludewig, N. Vandewalle, Europhys. Lett. 99, 40001 (2012)

[16] J. Kollmer, A. Sack, M. Heckel, F. Zimber, P. Müller, M.N. Bannerman, T. Pöschel, AIP Conference Proceedings 1542, 811 (2013)

[17] M. Noirhomme, F. Ludewig, N. Vandewalle, E. Opsomer, Phys. Rev. E 95, 022905 (2017)

[18] A. Sack, K. Windows-Yule, M. Heckel, D. Werner, T. Pöschel, Granular Matter 22, 54 (2020)

[19] T. Pöschel, T. Schwager, Computational Granular Dynamics (Springer-Verlag, 2005)

[20] D. Antypov, J.A. Elliott., Europhys. Lett. 94, 50004 (2011)

[21] Q.L. Wu, M.Y. Hou, L. Yang, W. Wang, G.H. Yang, K.W. Tao, L.W. Chen, S. Zhang, Chin. Phys. B 29, 054502 (2020)

[22] M. Adachi, P. Yu, M. Sperl, npj Microgravity 5, 19 (2019)

[23] D. Puzyrev, K. Harth, T. Trittel, R. Stannarius, Microgravity Sci. Technol. 32, 897 (2020)

[24] A. Sack, M. Heckel, J.E. Kollmer, F. Zimber, T. Pöschel, Phys. Rev. Lett. 111, 018001 (2013) 